\title{
Physiologie und Pathophysiologie der Venen
}

\author{
Federico Tatò
}

Anatomisch werden die Venen in ein oberflächliches und ein tiefes Venensystem unterteilt. Die tiefen Venen folgen in ihrem Verlauf den Arterien, während die epi- und intrafaszial verlaufenden oberflächlichen Venen von den Arterien unabhängig sind. Die peripher netzförmig angelegten oberflächlichen Venen vereinigen sich zu größeren Hauptstämmen, wie der V. saphena magna und parva, und münden an bestimmten anatomischen Fixpunkten in das tiefe Venensystem. Zusätzlich sind beide Venensysteme durch Brückenvenen, den Vv. perforantes, miteinander verbunden ( $\triangleright$ Kap. 76, „Anatomie der Gefäße: Untere Extremität").

Im venösen Niederdrucksystem tragen verschiedene Mechanismen zum herzgerichteten Blutfluss bei. Im Liegen sind die arteriovenöse Druckdifferenz und die abdominodiaphragmale Sogwirkung maßgeblich. Im Stehen steigt der Druck in den distalen Beinvenen auf $60-90 \mathrm{mmHg}$, und der Blutrücktransport aus den Beinvenen muss gegen die Schwerkraft erfolgen. Die Voraussetzung hierfür ist eine funktionierende Muskelfaszienpumpe. $\mathrm{Zu}$ diesem Zweck sind die Venen mit bikuspiden Klappen versehen, die nur eine herzgerichtete Flussrichtung erlauben. Entsprechend der im Stehen distal höheren Druckbelastung nimmt die Dichte der Venenklappen nach peripher zu, während die V. cava, die V. iliaca communis und die Viszeralvenen in der Regel klappenfrei sind. Die Funktion dieser Muskelpumpe setzt sowohl intakte Venenklappen als auch eine regelrechte Funktion der Beinmuskulatur voraus. Sowohl Schädigungen des Klappenapparates als auch Lähmungen oder sonstige Einschränkungen der Beinmotilität spielen daher eine wichtige Rolle für die Pathophysiologie der chronischvenösen Insuffizienz.
Das venöse System ist zusätzlich auch der wichtigste Blutspeicher des Körpers. Die extrathorakalen Venen enthalten $50-60 \%$ des Blutvolumens. Anders als in den Arterien bewirken geringe Druckänderungen im venösen System große Volumenschwankungen. Der vorwiegend über das sympathische Nervensystem zentral regulierte Venentonus spielt daher eine wichtige Rolle bei der Steuerung der für den Kreislauf verfügbaren Blutmenge.

Entsprechend dem Niederdruck des venösen Kreislaufs sind die Venen dünnwandig und nur schwach muskulär verstärkt. Sie sind zart und sehr vulnerabel. Tunica intima und Tunica media bleiben durch kollagene Fasern verbunden, so dass eine instrumentelle Trennung (Desobliteration) unmöglich ist. Arteriosklerotische Veränderungen kommen an Venen nicht vor. Um trotz ihrer Wandschwäche ihr Lumen zu erhalten, ist die Adventitia durch elastische Fasern mit dem Interstitium verbunden (Gewebefixierung). Dies bleibt bei der Präparation der Gefäße zu beachten, bei Denudierungen ist ein Venenkollaps mit nachfolgender Thrombose oftmals das Resultat.

\section{Literatur}

Caggiati A et al (2002) Nomenclature of the veins of the lower limbs: an international interdisciplinary consensus statement. J Vasc Surg $36: 416-422$

Hoffmann U, Tatò F (2013) Periphere Zirkulation. In: Siegenthaler W, Blum HE (Hrsg) Klinische Pathophysiologie, 9. Aufl. Thieme, Stuttgart/New York, S 695-729

Meissner MH et al (2007) The hemodynamics and diagnosis of venous disease. J Vasc Surg 46:4-24

Partsch H (2009) Varicose veins and chronic venous insufficiency. Vasa 38:293-301

F. Tatò $(\bowtie)$

Gefäßpraxis im Tal, München, Deutschland

E-Mail: f.tato@t-online.de 